\title{
Resveratrol Enhances the Bioavailability of Fexofenadine in Healthy Human Male Volunteers: Involvement of P-Glycoprotein Inhibition
}

\section{Satish Kumar Bedada1, Sudhakar Akul Yakkanti ${ }^{2}$ and Prasad Neerati ${ }^{1 *}$}

${ }^{1}$ DMPK and Clinical Pharmacology Division. Department of Pharmacology, University College of Pharmaceutical Sciences, Kakatiya University, Warangal, Telgana, India ${ }^{2}$ Cell Signaling and Matrix Biology Center, Foster City, CA, 94404, USA

\begin{abstract}
Objective: The purpose of the present study was to assess the influence ofresveratrol onP-glycoprotein mediated drug disposition in humans using fexofenadine as a P-glycoprotein substrate.

Methods: A non-blinded, an open label crossover study was conducted in twelve healthy male volunteers aged between 26 and 31 years. A single dose of fexofenadine hydrochloride $120 \mathrm{mg}$ was given to volunteers during control phase and treatment phases. A single dose of resveratrol $500 \mathrm{mg}$ was given to volunteers once daily for period of 10 days. The blood samples were collected at predetermined time intervals during control and treatment phases. The plasma samples containing fexofenadine hydrochloridewere analyzed by LC-MS/MS. The pharmacokinetic parameters were computed by non-compartmental method and the mean pharmacokinetic parameter differences during control and treatment phases were assessed.
\end{abstract}

Results: Treatment with resveratrol significantly increased thearea under the plasma concentration-time curve (AUC) and maximum plasma concentration $\left(\mathrm{C}_{\max }\right)$ offexofenadine to $76.7 \%(2520.92 .48$ versus 4454.48 ng.h $/ \mathrm{mL}$ ) and $65.2 \%$ (415.08 versus $685.58 \mathrm{ng} / \mathrm{mL}$ )respectively when compared to control phase. On other hand, apparentoral clearance $(\mathrm{CL} / \mathrm{F})$ and apparent volume of distribution $(\mathrm{Vd} / \mathrm{F})$ of fexofenadine were significantly decreased by $42.6 \%$ ( 49.46 versus $28.37 \mathrm{~L} / \mathrm{h}$ ) and $42.1 \%$ (591.73 versus $342.62 \mathrm{~L}$ ) respectively. However, there was no significant change was observed in $\mathrm{T}_{1 / 2}, \mathrm{~K}_{\mathrm{el}}$ and $\mathrm{T}_{\max }$ of fexofenadine upon treatment with resveratrol when compared to control phase.

Conclusion: The results of the present study showed that multiple doses of resveratrolenhanced the bioavailability of fexofenadine probably by the inhibition of P-glycoprotein mediated drug efflux in humans.

Keywords: Fexofenadine; Resveratrol; P-glycoprotein; Pharmacokinetics; Bioavailability

\section{Introduction}

Resveratrol (RSV) (3,4',5-trihydroxystilbene) is a naturally occurring polyphenolic phytoalexin is naturally present in fruits, vegetables, grape skins and especially in red wine. RSV possessesdiverse biochemical and physiological properties including anti-inflammatory, immune modulatory activities as well as wide range of health benefits ranging from chemoprevention to cardio protection [1]. It is produced by the plants in response to stress, injury, ultraviolet irradiation and fungal infection as part of their defense mechanism. In addition, it is synthesized by grapes in response to fungal infections and foundin red wine at levels between 1 and $10 \mu \mathrm{M}[2]$.

RSV modulates the synthesis of hepatic apolipoprotein, lipids, inhibits platelet aggregation and eicosanoid production in human platelets and neutrophils [3]. Moreover, RSV inhibits events associated with tumor initiation, promotion and progression [4]. It has been reported that RSV increased the accumulation of daunorubicin (a $\mathrm{P}$-glycoprotein substrate) in $\mathrm{KB}-\mathrm{C} 2$ cells in a concentration dependent manner by inhibiting P-gp (P-glycoprotein) [5]. Additionally, RSV has been found to reversethe multidrug resistance in KBv200 cells by inhibiting the multidrug resistant gene expression [6]. It has been previously reported that RSV enhanced the chemosensitivity of doxorubicin in multidrug resistant human breast cancer cells by increasing the cellular influx of doxorubicin [7]. In addition, RSV has been found to potentiate the cytotoxic activity P-gp substrates such as docetaxel and doxorubicin due to enhanced intracellular levels by inhibition of P-gp and down regulation of mdr1 gene [8]. Furthermore, in vivo studies in rats demonstrated that RSV was able to increase the bioavailability of P-gp substratesthrough the inhibition of P-gp mediated drug efflux $[9,10]$. Since RSV is a P-gp inhibitor and may potentially inhibit efflux transporter responsible for the poor absorption of P-gp substrates, the influence of RSV treatment on the pharmacokinetics of known P-gp substrate, fexofenadine hydrochloride (FEX) in healthy volunteers is the subject of current investigation.

FEX is a non-sedating antihistamine drug which is indicated for treatment of seasonal allergic rhinitis and chronic urticaria [11]. It has been demonstrated that after oral administration of $\left[{ }^{14} \mathrm{C}\right]$ FEX to healthysubjects, $92 \%$ of the total dose was recovered, $12 \%$ in urine and $80 \%$ in the feces in unchanged form [12]. It has been reported that FEX is a $\mathrm{P}$-gp substrate and the low passive membrane permeability of FEX makes the absorption of this drug to be P-gp related. The low

*Corresponding author: Neerati Prasad, Assistant Professor, DMPK and Clinical Pharmacology Division, Department of Pharmacology, University College of Pharmaceutical Sciences, Kakatiya University, Warangal-506009, Telgana, India, Tel: +91 9494812120; Fax: +91 8702453508; E-mail: prasadneerati@gmail.com

Received August 17, 2014; Accepted August 25, 2014; Published October 03, 2014

Citation: Bedada SK, Yakkanti SA, Neerati P (2014) Resveratrol Enhances the Bioavailability of Fexofenadine in Healthy Human Male Volunteers: Involvement of P-Glycoprotein Inhibition. J Bioequiv Availab 6: 158-163. doi:10.4172/jbb.1000198

Copyright: (c) 2014 Bedada SK, et al. This is an open-access article distributed under the terms of the Creative Commons Attribution License, which permits unrestricted use, distribution, and reproduction in any medium, provided the original author and source are credited. 
intestinal permeability and almost no metabolism of FEX in vivo make it a suitable model substrate to evaluate the role of P-gp in drug efflux. The pharmacokinetic profile of FEX primarily depends on the activity of P-gp, an efflux transporter which is expressed in the small intestinemucosa, hepatocytes, kidney and blood-brain barrier [13,14].

In this study, we hypothesized that if RSV acts as aninhibitor of P-gp mediated efflux, possibly in the intestine, it will increase the bioavailability of FEX, a P-gpsubstrate. To our knowledge, there are no available data thatshow any inhibitory effect of RSV on P-gp mediateddisposition in humans. Therefore, we investigated thepossible effect of RSV on P-gp mediated efflux usingFEX as a P-gp substrate in healthy male volunteers.

\section{Materials and Methods}

\section{Materials}

FEX and Diphenhydramine (DPH) were obtained from Matrix Labs Limited (Hyderabad, India). RSV $500 \mathrm{mg}$ capsules were procured from Zenith Nutritions (Banglore, India). FEX $120 \mathrm{mg}$ tablets (Allegra) were purchased from Aventis Pharma Ltd (Mumbai, India). Solvents used for quantitative analysis (Merck, India) and all other chemicals, reagents which were used in the study are of analytical grade.

\section{Subjects}

Twelve healthy male volunteers with an age of $28.17 \pm 1.59$ (range 26-31) years, weight of $68.3 \pm 7.95$ (range 52-82) kg, height of 173.2 \pm 7.33 (range 165-188) $\mathrm{cm}$ and body mass index (BMI) of $22.76 \pm$ 2.02 (range 17.99-24.95) were participated in the study (Table 1). All participants gave their written informedconsent prior to the study. The participants were confirmed as healthy by their medical history, physical examination androutine laboratory tests before enrollment. All the subjectswere nonsmokers, ate a normal diet and were not taking anyherbal dietary supplements. The subjects were instructed toabstain from taking any medication for at least 2 weeks priorto and during the study period. All the subjects were asked toabstain from alcohol, caffeine-containing beverages, tea andfruit juices during the study period.

\section{Study design}

The study protocol was approved by the institutional human ethical committee (UCPSC/KU/BA/2012-08) of University College of pharmaceutical sciences, Kakatiya University, Warangal, India. A nonblinded, open-label, two-way crossover studywas performed. The study phases were divided into control andtreatment separated by a 2 -week washoutperiod. The general study design was identical in control and treatment phases. The participants were administered with oral RSV $500 \mathrm{mg}$ capsules oncedaily for duration of 10 days (Zenith Nutritions, Banglore, India). A single oral dose of FEX $120 \mathrm{mg}$ (Allegra, Aventis Pharma Ltd, India) was administered during control phase and on eleventh day after RSV treatment with $240 \mathrm{~mL}$ water under fasting conditionsof more than $10 \mathrm{~h}$. The doses of RSV and FEX were selected based on the marketed formulations. The drugs used in this study were given in the sitting position and the subjects remained seated for $4 \mathrm{~h}$ after administration of FEX. Standardizedmeals were given at 4 and 10 $\mathrm{h}$ after dosing. Blood samples were drawn immediately before as well as $0.5,1,1.5,2,2.5,3,4,6,8,12,24,36$ and $48 \mathrm{~h}$ after FEX administration. Blood samples were collected in heparinized vacutainers and centrifuged at $4,000 \mathrm{rpm}$ for $15 \mathrm{~min}$ and theseparated plasma samples were stored at $-70^{\circ} \mathrm{C}$ until the assaywas performed.

\section{Determination of fexofenadine hydrochloride concentration inplasma}

FEX concentration in plasma samples wasassayed using LCMS/MS method [15] by employing protein precipitation extraction method. Aliquots of plasma samples $(100 \mu \mathrm{L})$ were added to $200 \mu \mathrm{L}$ acetonitrile with DPH $(4 \mathrm{ng} / \mathrm{mL})$ as an internal standard, then mixed in a vortex mixer for $3 \mathrm{~min}$ and centrifuged at 14,000 rpmfor $8 \mathrm{~min}$. Next pipette out $150 \mu \mathrm{L}$ of supernatant, then $5 \mu \mathrm{L}$ aliquot was injectedinto the analytical column. The plasma concentration of FEX was analyzed by LC-MS/MS with Agilent 1200 quaternary pump, auto sampler with thermostat, column oven, online degasser and triple quadrupole mass spectrometer (Mass hunter software version B.03.01) with multimode source (Agilent Technologies, USA). A Symmetry C18 reverse phase column $(75 \mathrm{~mm} \times 4.6 \mathrm{~mm}$, i.d. $3.5 \mu \mathrm{m})$ and the mobile phase (acetonitrile: $10 \mathrm{mM}$ ammonium acetate: $0.1 \%$ formic acid $=70: 30$ ) at a flow rate of $0.2 \mathrm{~mL} / \mathrm{min}$ were used. FEX was monitored in positive ion mode with the transition of $\mathrm{m} / \mathrm{z} 502.3$ to $\mathrm{m} / \mathrm{z} 466.2$ and DPH with the transition of $\mathrm{m} / \mathrm{z} 256.2$ to $\mathrm{m} / \mathrm{z} 167.0$ respectively. The lower limit of quantification for FEX was $0.5 \mathrm{ng} / \mathrm{mL}$, and the assay range used was 0.5-1000 ng/mL. Correlation coefficient for FEX calibration curves was 0.998.The intra-day and inter-day coefficient of variations for the low andhigh quality control samples were less than $15 \%$.

\section{Pharmacokinetic analysis}

The pharmacokinetics of FEXwas estimated usingnoncompartmental method using Phoenix WinNonlin 6.2.1 software (Certara, Pharsight Corporation, USA). Themaximum plasma concentration $\left(\mathrm{C}_{\max }\right)$ and the time taken to reach $\mathrm{C}_{\max }$ is $\mathrm{T}_{\max }$ were estimated directly from the observedplasma concentration-time data. The elimination rate constant $\left(\mathrm{K}_{\mathrm{el}}\right)$ was determined by linear regression analysis of thelog-linear part of the plasma concentration-time curve. Thetotal area under the plasma concentration-time curve (AUC)was calculated using the linear trapezoidal rule. The AUCfrom 0 to infinity $\left(\mathrm{AUC}_{\text {inf }}\right.$ ) was calculated as $\mathrm{AUC}_{\text {inf }}=\mathrm{AUC}+\mathrm{Ct} / \mathrm{K}_{\mathrm{el}}$ (where $\mathrm{Ct}$ was the last plasma concentration measured). The half-life $\left(\mathrm{T}_{1 / 2}\right)$ of FEX was calculated using $\mathrm{T}_{1 / 2}=\ln 2 / \mathrm{K}_{\mathrm{el}}$. The apparent oral clearance $(\mathrm{CL} / \mathrm{F})$ of FEX was calculated as $\mathrm{CL} / \mathrm{F}=\mathrm{dose} / \mathrm{AUC}_{\text {inf }}$ and the apparent volume of distribution $(\mathrm{Vd} / \mathrm{F})$ was calculated using $\mathrm{Vd} / \mathrm{F}=\mathrm{CL} / \mathrm{F} / \mathrm{\lambda z}$.

\section{Statistical analysis}

The results are expressed as the mean \pm SD. Thepharmacokinetics of FEX between the control and treatment phaseswere compared to each other using a paired $t$-test (twotailed) or the Wilcoxon signed

\begin{tabular}{|c|c|c|c|c|}
\hline Subject & Age (Yrs) & Height $\mathbf{( c m )}$ & Weight $\mathbf{( K g )}$ & $\mathbf{B M I} \mathbf{( k g} / \mathbf{m}^{\mathbf{2}} \mathbf{)}$ \\
\hline 1 & 28 & 175 & 69 & 22.53 \\
\hline 2 & 26 & 185.5 & 82 & 23.83 \\
\hline 3 & 26 & 170 & 52 & 17.99 \\
\hline 4 & 28 & 175 & 75 & 24.49 \\
\hline 5 & 28 & 167.5 & 65 & 23.17 \\
\hline 6 & 27 & 167.5 & 65 & 23.17 \\
\hline 7 & 29 & 175 & 63 & 20.57 \\
\hline 8 & 30 & 167.5 & 63 & 22.45 \\
\hline 9 & 31 & 175 & 76 & 24.82 \\
\hline 10 & 28 & 167.5 & 70 & 24.95 \\
\hline 11 & 27 & 188 & 75 & 21.22 \\
\hline 12 & 30 & 165 & 65 & 23.88 \\
\hline Mean & 28.17 & 173.21 & 68.33 & 22.76 \\
\hline SD & 1.59 & 7.33 & 7.95 & 2.02 \\
\hline
\end{tabular}

Table 1: Demographic characteristics of healthy male volunteers. 
Citation: Bedada SK, Yakkanti SA, Neerati P (2014) Resveratrol Enhances the Bioavailability of Fexofenadine in Healthy Human Male Volunteers: Involvement of P-Glycoprotein Inhibition. J Bioequiv Availab 6: 158-163. doi:10.4172/jbb.1000198

rank sum test after normalitytest using Kolmogorov-Smirnov test. All the dataobtained were analyzed using Graph Pad Prism 5.1 software (GraphPad Software Inc., San Diego, CA, USA). A $P<0.05$ was considered statistically significant. To determine the possibility of druginteraction between RSV and FEX, wecompared calculated individual pharmacokinetic parameters and their ratios (test/ reference) using log-transformed data;their geometric means and $90 \%$ confidence intervals (CIs) were analyzed. The resulting confidence limits were transformed by exponentiationand reported on the original measurement scale. The statistical limits were set at 0.80-1.25.

\section{Results}

The pharmacokinetic parameters and mean plasma concentrationtime profiles of FEX after a 10-day treatment with RSV are shown in Table 2 and Figure 1, respectively. During the study period, no serious adverse effects related to the drugs were reported. When we measured the plasma FEX concentrations after a single oral dose of $120 \mathrm{mg}$ FEX, those of RSV phase were increased compared to those of the control phase (Figure 1). The mean AUC of fexofenadine was increased by $76.7 \%$ after RSV treatment $(2520.92 \pm 516.92$ versus $4454.48 \pm 1238.27$ ng.h/mL, $\mathrm{P}<0.05)$ as compared to control phase. In addition, RSV increased the mean $\mathrm{C}_{\max }$ value by $65.2 \%$ compared to that of the control phase $(415.08 \pm 67.63$ versus $685.58 \pm 184.24 \mathrm{ng} / \mathrm{mL}, P<0.05)$. The mean value of $\mathrm{CL} / \mathrm{F}$ and $\mathrm{Vd} / \mathrm{F}$ were decreased significantly by $42.6 \%(49.46$ \pm 12.27 versus $28.37 \pm 7.03 \mathrm{~L} / \mathrm{h}, P<0.05)$ and $42.1 \%(591.73 \pm 197.25$ versus $342.62 \pm 108.56 \mathrm{~L}, P<0.05)$ respectively after RSV treatment as compared to control phase. The AUC andC $\mathrm{m}_{\max }$ of FEX were increased where as $\mathrm{CL} / \mathrm{F}$ and $\mathrm{Vd} / \mathrm{F}$ were decreased in all individualsupon RSV treatment as compared to control phase (Figure 2). However, there was no significant change was observed in $\mathrm{T}_{1 / 2}, \mathrm{~T}_{\max }$ and $\mathrm{K}_{\mathrm{el}}$ of FEX between RSV treatment and control phases. The $90 \%$ CI for the ratio of geometric means (RSV treatment phase:control phase) for AUC, $\mathrm{C}_{\text {max }} \mathrm{CL} / \mathrm{F}$ and $\mathrm{Vd} / \mathrm{F}$ were fell outside the interval (0.8-1.25) but in case $\mathrm{T}_{1 / 2}, \mathrm{~T}_{\max }$ and $\mathrm{K}_{\mathrm{el}}$ fell within the interval (Table 2) which indicates the interaction between RSV and FEX, consequently the pharmacokinetics of FEX were altered upon RSV treatment.

\section{Discussion}

P-gp is an adenosine triphosphate (ATP)-dependentmultidrug transporter expressed mainly on theapical membrane of the intestine. P-gp has been reported topromote the elimination of many drugs into the intestinallumen, thereby limiting their gastrointestinal absorption.Pgp can also reduce the intracellular concentrations of manycytotoxic anti-tumor drugs and is involved in multidrug resistancein tumor cells [16]. Recently inhibition of this transporter protein was implicated as a mechanism underlying certain drug-phytochemical interactions. In the clinical setting, familiarity with known inhibitors of P-gp, together with knowledge of the role of P-gp in determining substrate pharmacokinetics, should increase awareness of potential adverse or desired effects when phytochemicals that interact with P-gp substrates.

\begin{tabular}{|c|c|c|c|c|}
\hline Pharmacokinetic parameters & Control phase & Treatment phase & Geometric mean ratio & $90 \% \mathrm{Cl}$ \\
\hline$C_{\max }(\mathrm{ng} / \mathrm{mL})$ & $415.08 \pm 67.63$ & $685.58 \pm 184.24^{*}$ & 1.61 & $1.38-1.88$ \\
\hline$T_{\max }(h)$ & $2.37 \pm 0.37$ & $2.41 \pm 0.36$ & 1.02 & $0.94-1.10$ \\
\hline $\operatorname{Ke}\left(h^{-1}\right)$ & $0.088 \pm 0.02$ & $0.087 \pm 0.02$ & 0.99 & $0.87-1.12$ \\
\hline$T_{1 / 2}(h)$ & $8.27 \pm 1.82$ & $8.48 \pm 2.15$ & 1.01 & $0.91-1.14$ \\
\hline$A U C_{\text {last }}(\mathrm{ng} \cdot \mathrm{h} / \mathrm{mL})$ & $2520.92 \pm 516.92$ & $4454.48 \pm 1238.27^{*}$ & 1.74 & $1.53-1.98$ \\
\hline$A U C_{\text {inf }}(\mathrm{ng} \cdot \mathrm{h} / \mathrm{mL})$ & $2541.65 \pm 527.18$ & $4512.33 \pm 1265.17^{*}$ & 1.75 & $1.54-1.99$ \\
\hline CL/F (L/h) & $49.46 \pm 12.27$ & $28.37 \pm 7.03^{*}$ & 0.56 & $0.50-0.64$ \\
\hline Vd/F (L) & $591.73 \pm 197.25$ & $342.62 \pm 108.56^{*}$ & 0.58 & $0.48-0.69$ \\
\hline
\end{tabular}

Pharmacokinetic data are expressed as mean $\pm \mathrm{SD}$. ${ }^{*} P<0.05$, compared with the control phase.

Table 2: Pharmacokinetic parameters of fexofenadine after a single-dose administration of $120 \mathrm{mg}$ fexofenadine hydrochloride in 12 healthy subjects, after 500 mg resveratro once daily for 10 days.

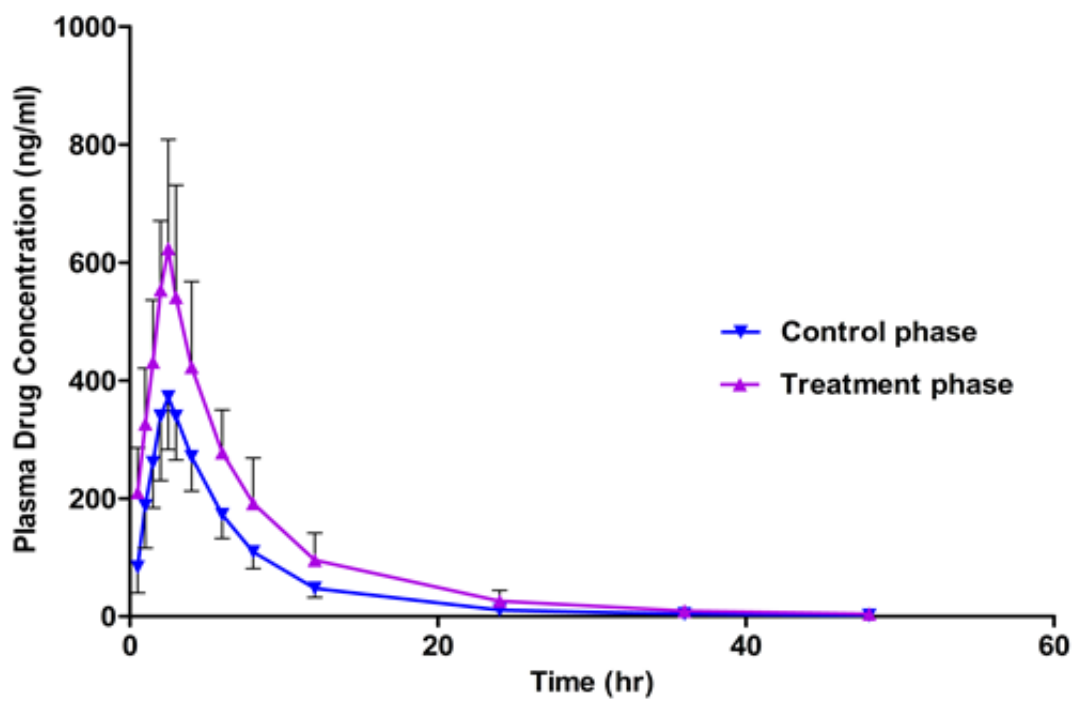

Figure 1: Mean plasma concentrations of fexofenadine in 12 healthy volunteers after a single 120 mg oral dose of fexofenadine hydrochloride, following a 10 -day treatment with resveratrol $500 \mathrm{mg}$ once daily. Values are shown as shown as the mean \pm SD. 
(A)

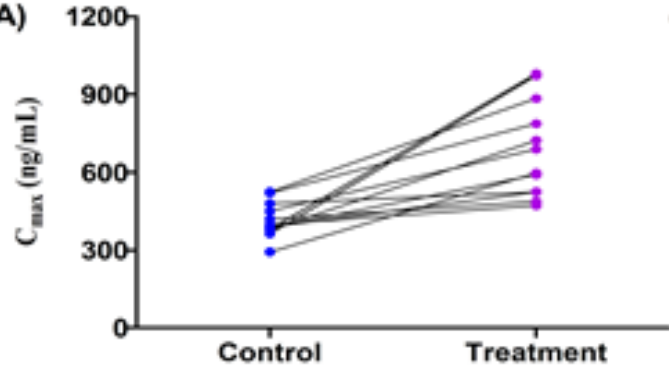

(C)

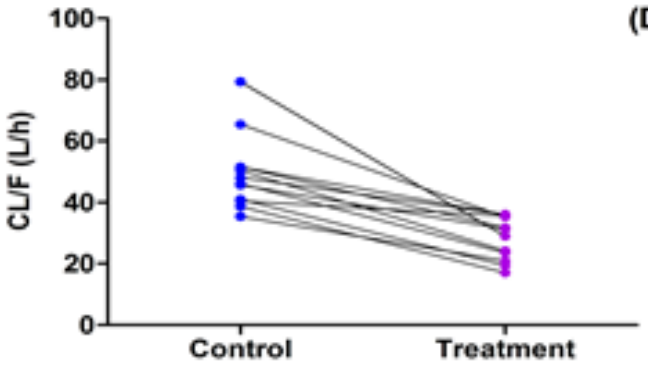

(B)

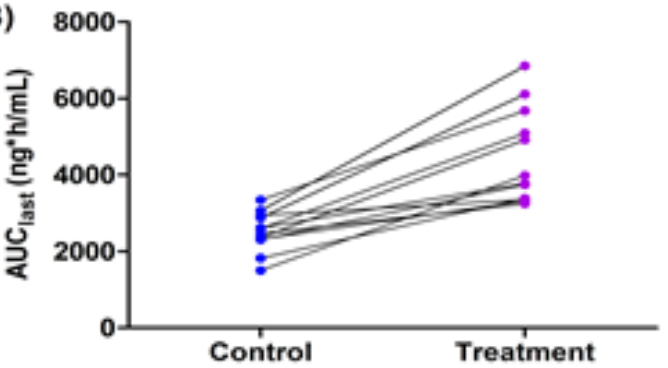

(D)

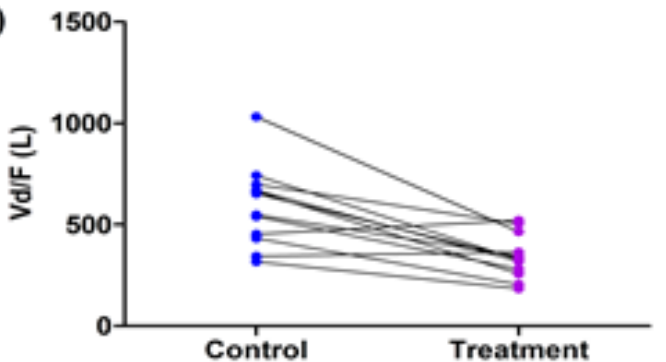

Figure 2: Individual peak plasma concentration (Cmax) (A), area under the concentration (AUC) (B), apparent oral clearance (CL/F) (C) and apparent volume of distribution $(\mathrm{Vd} / \mathrm{F})(\mathrm{D})$ of fexofenadine in 12 healthy volunteers after a single $120 \mathrm{mg}$ oral dose of fexofenadine hydrochloride, following a 10-day treatment with resveratrol $500 \mathrm{mg}$ once daily.

In this study, we assessed the effect of RSV on the pharmacokinetics of FEX, a substrate of P-gp in humans and found that RSV affected the pharmacokinetics of FEX and increased its bioavailability. To assess the inhibitory effect of RSV on P-gp in humans, we used FEX as a $\mathrm{P}$-gp substrate [17]. RSV treatment significantly increased the $\mathrm{C}_{\max }$ and AUC of FEX to $65.2 \%$ and $76.6 \%$ respectively as compared to control phase. On the other hand, CL/F and Vd/F of FEX were significantly decreased to $42.6 \%$ and $42.1 \%$ respectively as compared to control phase. However,there was no significant change was observed in $T_{1 / 2}$ $\mathrm{T}_{\max }$ and $\mathrm{K}_{\mathrm{el}}$ of FEX upon RSV treatment when compared to control phase. The interaction between RSV and FEX was also assessed by geometric mean ratios and $90 \%$ CI. The $90 \%$ CI for the ratio of geometric means for AUC, $\mathrm{C}_{\max }, \mathrm{CL} / \mathrm{F}$ and $\mathrm{Vd} / \mathrm{F}$ were fell outside the interval (0.8-1.25) which indicates the significant difference in mean values of $\mathrm{AUC}, \mathrm{C}_{\max }, \mathrm{CL} / \mathrm{F}$ and $\mathrm{Vd} / \mathrm{F}$ between control and treatment phases. On the other hand, $90 \% \mathrm{CI}$ for the ratio of geometric means for $\mathrm{T}_{1 / 2}, \mathrm{~T}_{\max }$ and $\mathrm{K}_{\mathrm{el}}$ fell within the interval (0.8-1.25) which indicates the insignificant difference in mean values of $\mathrm{T}_{1 / 2}, \mathrm{~T}_{\max }$ and $\mathrm{K}_{\mathrm{el}}$ between control and treatment phases. These findings provide in vivo evidence that RSV might increase the bioavailability of FEX via the inhibition of $\mathrm{P}$-gp mediateddrug efflux during the absorption phase in the intestine. Likewise, other P-gp inhibitors such as itraconazole [18-20], ritonavir [21] exhibited similar inhibitory effects on the pharmacokinetics of FEX, namely an increase in $\mathrm{C}_{\max }$ and AUC, decrease in $\mathrm{CL} / \mathrm{F}$ and $\mathrm{Vd} / \mathrm{F}$. On the other hand, in the case of P-gp inducers such as St. John's wort [22], rifampin [23] and carbamazepine [24], the CL/F and $\mathrm{Vd} / \mathrm{F}$ of FEX were reported to have been increased while the $\mathrm{C}_{\max }$ and AUC were decreased. Furthermore, it has been reported that P-gp was the main transport protein responsible for FEX transport and the in vitro FEX transport was inhibited by various $\mathrm{P}$-gp inhibitors such as ketoconazole, verapamil, erythromycin and ritonavir in the Caco-2 cell model $[25,26]$. Considering that $\mathrm{T}_{1 / 2}$ and $\mathrm{T}_{\max }$ were not changed significantly by RSV, the results of the presentstudy suggest that RSV affects P-gp expressed in thesmall intestine instead of the kidney and liver.

In this study we assessed whether RSV could act as an inhibitor of P-gp mediateddrug efflux. Previously it has been reported that RSV exerted an inhibitory effect on P-gp mediated efflux in vitro resulted in increased intracellular accumulation of daunorubicin [5]. In addition, an in vitro study has demonstrated that RSV enhanced the chemosensitivity of doxorubicin by increasing the cellular influx of doxorubicin [7]. Additionally, RSV has been found to potentiate the cytotoxic activity of docetaxel and doxorubicin due to enhanced intracellular levels by inhibition of P-gp [8]. Further, in vivo studies in rats demonstrated that RSV was able to increase the bioavailability of P-gp substratesthrough the inhibition of P-gp mediated drug efflux $[9,10]$. It has been reported that RSV exertedan inhibitory effect on drugmetabolizingenzymeCYP3A4 in vitro [27], similarly RSV exhibited inhibitory effect on drugmetabolizingenzymes including CYP3A4, CYP2D6, and CYP2C9 in vivo in human volunteers [28]. Therefore, it is possible a priori to assume that the interaction between RSV and FEX might result from the inhibition of RSV on the metabolism of FEX. However, to our knowledge, FEX is not a substrate of these enzymes and it has been revealed that FEX itself is not further metabolized $[29,12]$. It has been previously reported that verapamil (a standard P-gp inhibitor) enhanced the bioavailability of FEX through P-gp inhibition [30]. Moreover, our results were comparable to previous work where quercetin substantially enhanced the bioavailability of FEX in healthy volunteers by inhibiting P-gpmediated drug efflux in the intestine [13]. Based on these findings, we suggest that RSV acts as an inhibitor of P-gpmediated efflux of FEX in humans.

Recently, it has been reported that in addition to P-gp,FEX is also a substrate of drug uptake transporters,such as organic anion transporter (OAT-3) and organicanion-transporting polypeptides (OATP)-A (OATP1A2) and OATP-B (OATP2B1) [12,17,31,32]. Dresser et al. [33] reported that grapefruit and apple juicesreduce FEX 
Citation: Bedada SK, Yakkanti SA, Neerati P (2014) Resveratrol Enhances the Bioavailability of Fexofenadine in Healthy Human Male Volunteers: Involvement of P-Glycoprotein Inhibition. J Bioequiv Availab 6: 158-163. doi:10.4172/jbb.1000198

bioavailability through the inhibitionof OATP1A2 which resulted in delay in $\mathrm{T}_{\max }$. However, the inhibition of uptaketransporters leads to a delay in $\mathrm{T}_{\max }$, but no significant difference wasobserved in $\mathrm{T}_{\max }$ between the RSV treatment and control phases. Furthermore, eventhough the disposition of FEX may be affected by OATP, there were no reports available regarding the RSV having the inhibitory effect on OATPmediated efflux. Therefore, the role of OATP in the interaction between RSV and FEX may be minor compared to thatof P-gp.

RSV could be used as a P-gp inhibitor like verapamil, itraconazole and ritonavir and it is having the potential increase the bioavailability of P-gp substrates like digoxin, talinolol including FEX.In addition, the intake of dietary supplements containing RSV may increase the absorption or oral bioavailability of FEX and can be useful to reduce the dose of FEX and possibly helpful in reducing the side effects of FEX. However, further studies should be carried out in patients to explore the RSV mediated P-gp interactions and the potential clinical application of dietary phytochemicals as potent P-gp modulators.

\section{Conclusions}

The results of the present study showed that multiple use of RSV substantially enhanced thebioavailability of FEX, probably by the inhibition of P-gpmediated drug efflux in humans. The inhibition of P-gp by dietary phytochemicals containing RSV may provide a novel approach for improving the absorption and exposure of low bioavailable drugs like anti viral and anti cancer drugs.Accordingly, much effort is currently being expanded toward identifying natural compounds from plant origins that inhibitP-gp and developing as P-gp modulators.

\section{Acknowledgements}

PN had support from University Grants Commission, New Delhi, India (F.36130/2009.SR) and start-up research funds of University College of Pharmaceutical Sciences at Kakatiya University, Warangal, AP, India, for the submitted work. SY was partly supported by FAMRI-062558 and RO1CA143128.

\section{References}

1. Kalantari H, Das DK (2010) Physiological effects of resveratrol. Biofactors 36 401-406.

2. Soleas GJ, Diamandis EP, Golberg DM (1997) Resveratrol: a molecule whose time has come? and gone? Clin Biochem 30: 91-113.

3. Bertelli AA, Giovanni L, Stradi R, Urien S, Tillement JP, et al. (1996) Kinetics of trans- and cis-resveratrol (3,40,5-trihydroxystilbene) after red wine oral administration in rats. Int J Clin Pharmacol Res 26: 77-81.

4. Jang M, Cai L, Udeani GO, Slowing KV, Thomas CF, et al. (1997) Cancer chemopreventive activity of resveratrol, a natural product derived from grapes. Science 275: 218-220

5. Nabekura T, Kamiyama S, Kitagawa S (2005) Effects of dietary chemopreventive phytochemicals on P-glycoprotein function. Biochem Biophys Res Commun 327: 866-870.

6. Quan F, Pan C, Ma Q, Zhang S, Yan L (2008) Reversal effect of resveratrol on multidrug resistance in KBv200 cell line. Biomed Pharmacother 62: 622-629.

7. Kim TH, Shin YJ, Won AJ, Lee BM, Choi WS, et al. (2014) Resveratro enhances chemosensitivity of doxorubicin in multidrug-resistant human breast cancer cells via increased cellular influx of doxorubicin. Biochim Biophys Acta 1840: 615-625.

8. Al-AbdAM, Mahmoud AM, El-Sherbiny GA, El-Moselhy MA, Nofal SM, et al. (2011) Resveratrol enhances the cytotoxic profile of docetaxel and doxorubicin in solid tumour cell lines in vitro. Cell Prolif 44: 591-601.

9. Choi JS, Choi BC, Kang KW (2009) Effect of resveratrol on the pharmacokinetics of oral and intravenous nicardipine in rats: possible role of P-glycoprotein inhibition by resveratrol. Pharmazie 64: 49-52.

10. Hong SP, Choi DH, Choi JS (2008) Effects of resveratrol on the pharmacokinetics of diltiazem and its major metabolite, desacetyldiltiazem, in rats. Cardiovasc Ther 26: 269-275.

11. Simpson K, Jarvis B (2000) Fexofenadine: a review of its use in the management of seasonal allergic rhinitis and chronic idiopathic urticaria. Drugs 59: 301-321

12. Tahara H, Kusuhara $\mathrm{H}$, Maeda K, Koepsell H, Fuse E, et al. (2006) Inhibition of oat3-mediated renal uptake as a mechanism for drug-drug interaction between fexofenadine and probenecid. Drug Metab Dispos 34: 743-747.

13. Kim KA, Park PW, Park JY (2009) Short-term effect of quercetin on the pharmacokinetics of fexofenadine, a substrate of P-glycoprotein, in healthy volunteers. Eur J Clin Pharmacol 65: 609-614.

14. Drescher S, Schaeffeler E, Hitzl M, Hofmann U, Schwab M, et al. (2002) MDR1 gene polymorphisms and disposition of the P-glycoprotein substrate fexofenadine. Br J Clin Pharmacol 53: 526-534.

15. Guo D, Zou J, Zhu Y, Lou S, Fan H, et al. (2010) Measurement of fexofenadine concentration in micro-sample human plasma by a rapid and sensitive LC-MS/ MS employing protein precipitation: application to a clinical pharmacokinetic study. Biomed Chromatogr 24: 335-341.

16. Matheny CJ, Lamb MW, Brouwer KR, Pollack GM (2001) Pharmacokinetic and pharmacodynamic implications of P-glycoprotein modulation. Pharmacotherapy 21: 778-796.

17. Cvetkovic M, Leake B, Fromm MF, Wilkinson GR, Kim RB (1999) OATP and P-glycoprotein transporters mediate the cellular uptake and excretion of fexofenadine. Drug Metab Dispos 27: 866-871.

18. Shon JH, Yoon YR, Hong WS, Nguyen PM, Lee SS, et al. (2005) Effect of itraconazole on the pharmacokinetics and pharmacodynamics of fexofenadine in relation to the MDR1 genetic polymorphism. Clin Pharmacol Ther 78: 191201.

19. Uno T, Shimizu M, Sugawara K, Tateishi T (2006) Lack of dose dependent effects of itraconazole on the pharmacokinetic interaction with fexofenadine. Drug Metab Dispos 34: 1875-1879.

20. Shimizu M, Uno T, Sugawara K, Tateishi T (2006) Effects of itraconazole and diltiazem on the pharmacokinetics of fexofenadine, a substrate of P-glycoprotein. Br J Clin Pharmacol 61: 538-544

21. van Heeswijk RP, Bourbeau M, Campbell P, Seguin I, Chauhan BM, et al. (2006) Time-dependent interaction between lopinavir/ritonavir and fexofenadine. $J$ ClinPharmacol 46: 758-767.

22. Wang Z, Hamman MA, Huang SM, Lesko LJ, Hall SD (2002) Effect of St John's wort on the pharmacokinetics of fexofenadine. Clin Pharmacol Ther 71: 414420.

23. Hamman MA, Bruce MA, Haehner-Daniels BD, Hall SD (2001) The effect of rifampin administration on the disposition of fexofenadine. Clin Pharmacol Ther 69: 114-121.

24. Yamada S, Yasui-Furukori N, Akamine Y, Kaneko S, Uno T (2009) Effects of the P-glycoprotein inducer carbamazepine on fexofenadine pharmacokinetics. Ther Drug Monit 31: 764-768.

25. Perloff MD, von Moltke LL, Greenblatt DJ (2002) Fexofenadine transport in Caco-2 cells: inhibition with verapamil and ritonavir. J Clin Pharmacol 42: 12691274.

26. Petri N, Tannergren C, Rungstad D, Lennernas H (2004) Transport characteristics of fexofenadine in the Caco-2 cell model. Pharm Res 21: 13981404.

27. Chan WK, Delucchi AB (2000) Resveratrol, a red wine constituent, is a mechanism-based inactivator of cytochrome P450 3A4. Life Sci 67: 3103-3112.

28. Chow HH, Garland LL, Hsu CH, Vining DR, Chew WM, et al. (2010) Resveratro modulates drug- and carcinogen-metabolizing enzymes in a healthy volunteer study. Cancer Prev Res 3: 1168-1175.

29. Ling KH, Leeson GA, Burmaster SD, Hook RH, Reith MK, et al. (1995) Metabolism of terfenadine associated with CYP3A (4) activity in human hepatic microsomes. Drug Metab Dispos 23: 631-636.

30. Yasui-Furukori N, Uno T, Sugawara K, Tateishi T (2005) Different effects of three transporting inhibitors, verapamil, cimetidine, and probenecid, on fexofenadine pharmacokinetics. Clin Pharmacol Ther 77: 17-23.

31. Nozawa T, Imai K, Nezu J, Tsuji A, Tamai I (2004) Functional characterization of $\mathrm{pH}$-sensitive organic anion transporting polypeptide OATP-B in human. J Pharmacol Exp Ther 308: 438-445. 
Citation: Bedada SK, Yakkanti SA, Neerati P (2014) Resveratrol Enhances the Bioavailability of Fexofenadine in Healthy Human Male Volunteers: Involvement of P-Glycoprotein Inhibition. J Bioequiv Availab 6: 158-163. doi:10.4172/jbb.1000198

32. Niemi M, Kivisto KT, Hofmann U, Schwab M, Eichelbaum M, et al. (2005) Fexofenadine pharmacokinetics are associated with a polymorphism of the SLCO1B1 gene (encoding OATP1B1). Br J Clin Pharmacol 59: 602-604.
33. Dresser GK, Kim RB, Bailey DG (2005) Effect of grapefruit juice volume on the reduction of fexofenadine bioavailability: possible role of organic anion transporting polypeptides. Clin Pharmacol Ther 77: 170-177. 